\title{
Pharyngeal gonorrhea presenting with isolated neck pain
}

\author{
Lourdes DelRosso ${ }^{1,2^{*}}$, Jennifer Smith ${ }^{1}$, Michael Harper $^{1}$, Romy Hoque $^{2}$ \\ ${ }^{1}$ Department of Family Medicine, Louisiana State University School of Medicine, Shreveport, USA; \\ *Corresponding Author: lordydel@yahoo.com \\ ${ }^{2}$ Department of Neurology, Louisiana State University School of Medicine, Shreveport, USA
}

Received 25 January 2013; revised 26 February 2013; accepted 5 March 2013

\section{ABSTRACT \\ We present a case of a 22-year-old man with isolated neck pain due to pharyngeal gonorrhea.}

Keywords: Pharynx; Gonorrhea; Neck Pain

\section{INTRODUCTION}

Gonorrhea is a common sexually transmitted disease (STD) that can involve many areas. We present a case of a 22-year-old man with isolated neck pain due to pharyngeal gonorrhea.

\section{CASE REPORT}

A 22-year-old African American man presents with one month history of right sided neck pain worsened by head turning to the right. He denies fever, chills, night sweats, productive cough, diarrhea, abdominal pain, weight loss, throat pain, dysphagia, odynophagia, headaches, skin rashes, focal weakness, or focal sensory changes. He reported having unprotected oral sex with men. He denies smoking tobacco, drinking alcohol, or use of illicit drugs. On physical examination his head was normocephalic and atraumatic with full range of motion in his neck. His throat was clear without exudates, adenotonsillar hypertrophy or soft palate erythema. No sensory deficits were noted in the face or neck. The remainder of the physical examination and neurological examination was normal.

Serum testing for human immunodeficiency virus (HIV) and reactive plasma reagent were negative. Throat cultures for chlamydia were negative, and positive for gonorrhea. With his positive pharyngeal gonorrhea testing, the patient revealed having oral sex with men for money. The patient was treated with ceftriaxone $250 \mathrm{mg}$ intramuscular injection, and azithromycin 1 gram orally. Extensive counseling regarding sexually transmitted diseases (STD) risks and prevention were provided. Transnasal flexible laryngoscopy revealed diffuse lymphoid hyperplasia in posterior nasopharynx and hypopharynx (Figure 1).

\section{DISCUSSION}

Gonorrhea is the second most common reported infection requiring partner notification in the United States with over 300,000 cases in 2011 [1]. Kent et al. found that gonorrhea most commonly affects non-urethral sites. Up to $64 \%$ of gonorrhea would be missed without rectal and pharyngeal screening with $36 \%$ of men infected in the pharynx alone and $28 \%$ of men infected in more than one site [2]. In adolescent women, $11 \%$ - 26\% of gonorrhea cases would be missed without pharyngeal culture

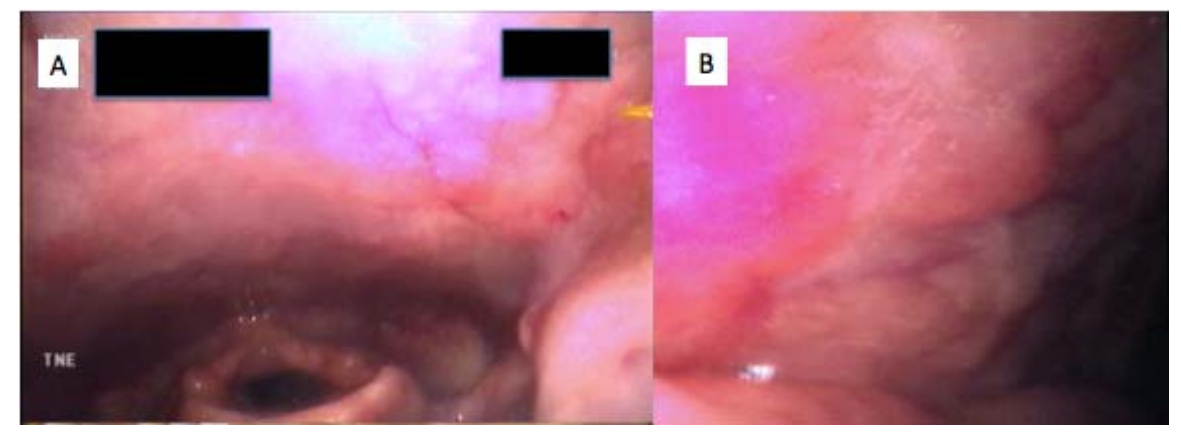

Figure 1. A: Diffuse lymphoid hyperplasia in a patient with pharyngeal gonorrhea presenting with isolated neck pain; B: Close-up of nodular hyperplasia in posterior pharyngeal wall. 
[3]. Positive predictors of pharyngeal gonorrhea infection are younger age and increased number of insertive oral sex partners [4]. Pharyngeal infections with gonorrhea are common in patients with HIV, and other STDs [5]. The Centers for Disease Control recommends treatment for both gonorrhea and chlamydia, given the prevalence of concurrent genital infection [1].

\section{CONCLUSION}

The neck pain in our patient resolved with treatment and was likely secondary to pharyngeal lymphoid hyperplasia. Isolated neck pain has also been reported in retropharyngeal abscess [6]. If the patients neck pain continued despite treatment, a computerized tomography of the neck would have been ordered to assess for retropharyngeal abscess.

\section{REFERENCES}

[1] CDC (2012) Update to CDC's sexually transmitted diseases treatment guidelines 2010: Oral cephalosporins no longer a recommended treatment for gonococcal infections. MMWR Morbidity and mortality weekly report, 61,
590-594.

[2] Kent, C.K., Chaw, J.K., Wong, W., Liska, S., Gibson, S., Hubbard, G., et al. (2005) Prevalence of rectal, urethral, and pharyngeal chlamydia and gonorrhea detected in 2 clinical settings among men who have sex with men: San Francisco, California, 2003. Clinical Infectious Diseases, 41, 67-74. doi: $10.1086 / 430704$

[3] Giannini, C.M., Kim, H.K., Mortensen, J., Marsolo, K. and Huppert, J. (2010) Culture of non-genital sites increases the detection of gonorrhea in women. Journal of Pediatric and Adolescent Gynecology, 23, 246-252. doi:10.1016/j.jpag.2010.02.003

[4] Morris, S.R., Klausner, J.D., Buchbinder, S.P., Wheeler, S.L., Koblin, B., Coates, T., et al. (2006) Prevalence and incidence of pharyngeal gonorrhea in a longitudinal sample of men who have sex with men: The explore study. Clinical Infectious Diseases, 43, 1284-1289. doi:10.1086/508460

[5] Mayor, M.T., Roett, M.A. and Uduhiri, K.A. (2012) Diagnosis and management of gonococcal infections. American Family Physician, 86, 931-938.

[6] Schott, C.K., Counselman, F.L. and Ashe, A.R. (2012) A pain in the neck: Non-traumatic adult retropharyngeal abscess. The Journal of Emergency Medicine, 44, 329331. 